\title{
Search For Wobbling Excitations In Hf Nuclei: Are The SD Bands Triaxial?
}

\author{
D. J. Hartley*, M. K. Djongolov ${ }^{\dagger}$, L. L. Riedinger ${ }^{\dagger}$, G. B. Hagemann**, R. V. F.

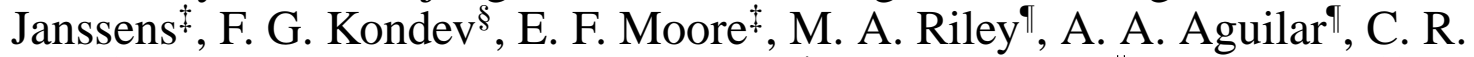 \\ Bingham $^{\dagger}$, D. B. Campbell ${ }^{\mathbb{I}}$, M. P. Carpenter", P. Chowdhury" , M. Cromaz ${ }^{\dagger \dagger}$, D. M. \\ Cullen $^{\ddagger \dagger}$, M. Danchev ${ }^{\dagger}$, G. D. Dracoulis ${ }^{\S \S}$, P. Fallon ${ }^{\dagger \dagger}$, J. Goon ${ }^{\dagger}$, R. A. Kaye ${ }^{\text {qIIII }}$, T. L. \\ Khoo $^{\ddagger}$, R. W. Laird ${ }^{* * *}$, T. Lauritsen ${ }^{\ddagger}$, A. O. Macchiavelli ${ }^{\dagger \dagger}$, B. McClain ${ }^{* * *}$, G. \\ Mukherjee $^{\ddagger \|}$, E. Ngijoi-Yogo $\|$, H. I. Park ${ }^{\dagger}$, G. Sletten**, S. K. Tandel ${ }^{\|}$, P. M. \\ Walker $^{\dagger \dagger \dagger}$ and Jing-ye Zhang ${ }^{\dagger}$ \\ ${ }^{*}$ Department of Physics, U.S. Naval Academy, Annapolis, MD 21402 \\ ${ }^{\dagger}$ Department of Physics and Astronomy, University of Tennessee, Knoxville, TN 37996 \\ ${ }^{* *}$ The Niels Bohr Institute, Blegdamsvej 17, DK-2100 Copenhagen, Denmark \\ ${ }^{\ddagger}$ Physics Division, Argonne National Laboratory, Argonne, IL 60439 \\ ${ }^{\S}$ Nuclear Engineering Division, Argonne National Laboratory, Argonne, IL 60439 \\ II Department of Physics, Florida State University, Tallahassee, FL 32306 \\ " Department of Physics, University of Massachusetts, Lowell, MA 01854 \\ ${ }^{\dagger}$ Physics Division, Lawrence Berkeley National Laboratory, Berkeley, CA 94720 \\ $\$$ Schuster Laboratory, University of Manchester, Manchester M13 9PL, United Kingdom \\ ${ }^{\S}$ Department of Nuclear Physics, Australian National University, Canberra, ACT 0200, Australia \\ TIII Department of Chemistry and Physics, Purdue University Calumet, Hammond, IN 46323 \\ ${ }^{* * *}$ Department of Physics, Trinity University, San Antonio, TX 78212 \\ ${ }^{\dagger \dagger}$ Department of Physics, University of Surrey, Guildford, Surrey GU2 5XH, United Kingdom
}

\begin{abstract}
Two Gammasphere experiments have been performed in order to establish the possible triaxial nature of strongly deformed (SD) bands in ${ }^{174} \mathrm{Hf}$. A lifetime measurement confirmed the large deformation of the four previously observed bands in this nucleus. In addition, a thin-target, high-statistics experiment was carried out to search for linking transitions between the SD bands. No such transitions, which represent an experimental signature for wobbling modes, were observed. Four new SD bands were found in ${ }^{174} \mathrm{Hf}$ together with a single SD band in ${ }^{173} \mathrm{Hf}$. These results indicate that the strongly deformed sequences of $N \approx 102 \mathrm{Hf}$ isotopes behave differently than the TSD bands found in Lu nuclei near $N=92$. The interpretation of these bands in terms of possible stable triaxial deformation is confronted with the experimental findings and UC predictions.
\end{abstract}

\section{INTRODUCTION}

Although triaxial deformation may play a role in describing various nuclear structure phenomena, establishing experimental evidence of stable triaxiality remains a challenge. Perhaps the best evidence of triaxial deformation is the observation of a "wobbling" mode since it is unique to a rotating asymmetric nucleus [1]. This mode is induced by the fact that the triaxial nucleus may rotate about each of its three axes, thus the rotation angular momentum vector may lie off the principal axis and precess around it. The degree to which this vector lies off axis is quantized and is characterized by the wobbling phonon $n_{w}$ [2]. Therefore, multiple rotational bands (with similar moments of inertia and alignments) may be observed in a nucleus based on the same configuration, but having different wobbling phonon values $\left(n_{w}=0,1,2, \ldots\right)$. Indeed, wobbling excitations have been confirmed in ${ }^{163} \mathrm{Lu}[3,4]$ for the band based on an $i_{13 / 2}$ proton, an orbital which increases the deformation in comparison with the other observed structures in this nucleus. For these reasons, the bands have been labeled triaxial strongly deformed (TSD). Linking transitions (with $\Delta I=1)$ are 


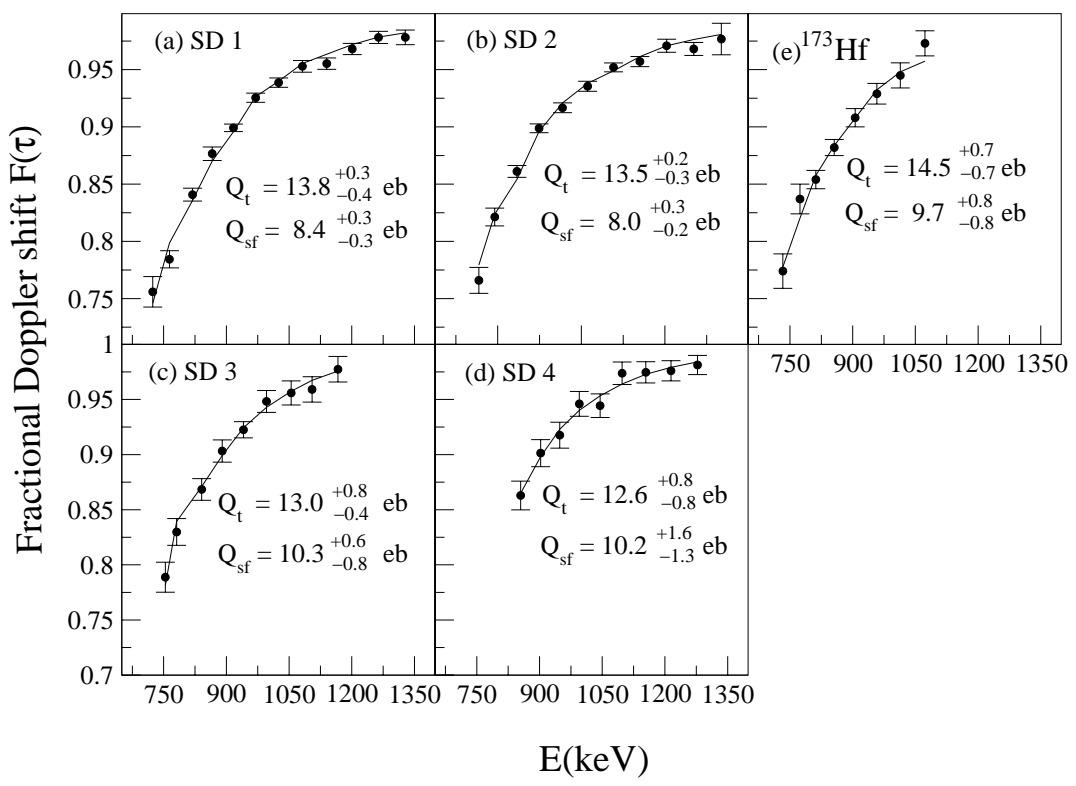

FIGURE 1. The data points represent the measured fractional Doppler shifts of transitions in the four previously known SD bands in ${ }^{174} \mathrm{Hf}[5]$ and the SD band in ${ }^{173} \mathrm{Hf}$. Results from the FITFTAU code are shown as solid lines, and the transition quadrupole $Q_{t}$ and sidefeeding $Q_{s f}$ moments from the fitting routine are given for each band.

observed between the $n_{w}=1$ wobbling excitation and the $n_{w}=0$ band, but these have a dominant $E 2$ character. This behavior is consistent with the predictions of wobbling bands by particle-rotor model calculations [3].

In ${ }^{174} \mathrm{Hf}$, we recently identified four bands with large moments of inertia, suggesting that they were strongly deformed (SD) [5]. Ultimate cranker (UC) calculations indicated that such structures may exist in TSD minima. Therefore, we have performed two experiments in an attempt to verify the possible triaxial nature of these bands. A lifetime measurement was performed to confirm the large (and similar) deformation of the bands. In addition, a high-statistics, thin-target experiment was run to search for linking transitions between the SD bands to provide evidence that some may be associated with wobbling excitations.

\section{EXPERIMENTAL DETAILS AND RESULTS}

The lifetime experiment was performed at the Lawrence Berkeley National Laboratory using the ${ }^{130} \mathrm{Te}\left({ }^{48} \mathrm{Ca}, 4 n\right)$ reaction at a beam energy of $200 \mathrm{MeV}$. The target consisted of a $850 \mu \mathrm{g} / \mathrm{cm}^{2}$ foil of ${ }^{130} \mathrm{Te}$ on a $26 \mathrm{mg} / \mathrm{cm}^{2}$ gold backing, and the Gammasphere [6] spectrometer detected the emitted $\gamma$ radiation. Over $3.5 \times 10^{9}$ five-fold or greater events were recorded and sorted into a Blue database [7]. Background subtraction of the double-gated spectra was performed with the method described by K. Starosta et al. [8]. The high-statistics, thin-target experiment utilized the same reaction; however, a higher beam energy of $205 \mathrm{MeV}$ was selected. The ${ }^{48} \mathrm{Ca}$ beam was provided by the ATLAS facility at Argonne National Laboratory and Gammasphere detected the $\gamma$ rays. Four ${ }^{130} \mathrm{Te}$ targets (ranging in thickness from 400 to $660 \mu \mathrm{g} / \mathrm{cm}^{2}$ ) were mounted onto a rotating wheel, where each had $\sim 500 \mu \mathrm{g} / \mathrm{cm}^{2}$ of gold on the front and $\sim 80 \mu \mathrm{g} / \mathrm{cm}^{2}$ of gold on the back. Approximately $2.6 \times 10^{9}$ four-fold or greater events were recorded and sorted into a coincidence cube as well as a hypercube.

In the analysis of the lifetime data it became clear that fully stopped transitions in the SD sequences were not present. Therefore, angle-dependent gating conditions that took into account the fractional Doppler shift $[F(\tau)]$ for a given energy were applied [9]. Initial $F(\tau)$ values were calculated assuming a quadrupole moment of $Q_{t}=11.4$ 
$e b$, which was measured for the strongest SD band in ${ }^{168} \mathrm{Hf}$ [10]. This procedure was repeated by varying $F(\tau)$ until the best possible enhancement of the transitions closest in energy to the gating $\gamma$ rays was achieved. These spectra provided the location of the gates for the next transitions in the sequence, and the final spectra at each angle were obtained by summing all such optimized double-gated spectra. The experimental fractional Doppler shift was then extracted from the centroid shift analysis by performing a linear regression of both $E_{0}$ and $F(\tau)$ in the expression $E(\theta)=E_{0}\left(1+\beta_{0} F(\tau) \cos (\theta)\right)$, where $E_{0}$ is the centroid at $90^{\circ}$ and $E(\theta)$ is the centroid at an angle $\theta$. The midtarget recoil velocity $\beta_{0}$ was calculated using SRIM 2003 [11]. In order to determine the quadrupole moment $Q_{t}$, computer simulations of the actual decay of the levels with the bands and their sidefeeding was performed with the code FITFTAU [12]. In the code, the following assumptions are implicit: 1) the $Q_{t}$ is constant within a given band, 2) the sidefeeding cascades have a common constant quadrupole moment $Q_{s f}$, and 3) the number of transitions that feed levels of the main cascade is proportional to the number of transitions above the state of interest. The sidefeeding sequence is assumed to have the same moment of inertia as the SD band and the feeding pattern is based upon the intensity pattern of the SD band, which was determined from the backed-target experiment.

Figure 1 displays the $F(\tau)$ data for the four previously known SD bands in ${ }^{174} \mathrm{Hf}$ along with the fits generated by FITFTAU. Large deformation has been established for all four bands with quadrupole moments ranging from $Q_{t}=12.6$ to $13.8 \mathrm{eb}$, see Figs. 1(a)-(d). The quoted errors are based solely on the uncertainty of determining the centroid energy of the peaks. An additional systematic error of $15-20 \%$ should be added to account for the uncertainties in the stopping powers. However, this does not affect the relative values as these bands were produced in the same experiment. In addition, a new SD band in ${ }^{173} \mathrm{Hf}$ was observed in the thin-target experiment (see below). After applying the same method to this band, a value of $Q_{t}=14.5_{-0.7}^{+0.7} \mathrm{eb}$ was determined for this ${ }^{173} \mathrm{Hf}$ sequence, see Fig. 1(e). Normal deformed structures are expected to have $Q_{t} \approx 7 \mathrm{eb}$, therefore, these measurements clearly indicate that all of these bands are, indeed, strongly deformed. Comparisons of these values with those predicted by the UC are discussed below.

The thin-target experiment allowed for the extension of the previously known bands to higher spin compared to the results reported in Ref. [5]. Spectra resulting from summing all possible triple-gate conditions of inband transitions for these bands are shown in Fig. 2(a)-(d). Bands 1 and 2 were both extended by three transitions, while five additional transitions were found for both bands 3 and 4. In addition, the 776 and 815-keV transitions in SD 4 were observed for the first time. Despite the higher statistics than those from our previous experiment [5], linking transitions to the normal deformed levels could not be identified; however, the strong coincidence with the ground-state sequence (denoted with filled circles in Fig.2) associates these structures with ${ }^{174} \mathrm{Hf}$. Four new, presumably strongly deformed, bands were also found in ${ }^{174} \mathrm{Hf}$ and are shown in Fig. 2(e)-(g). Similar to the four previously known SD bands, SD 5-8 could not be linked with the normal deformed levels, but once again, their coincidence with the ${ }^{174} \mathrm{Hf}$ ground-state band suggests that they are associated with this nucleus. Finally, a single SD band was observed in ${ }^{173} \mathrm{Hf}$ and is displayed in Fig. 2(i). One may note the difference in the low-energy $(<700 \mathrm{keV})$ portion of Fig. 2(i) in comparison with the other eight spectra. The majority of these low-energy $\gamma$ rays are observed in two sequences of ${ }^{173} \mathrm{Hf}$. The quadrupole moments of the new bands in ${ }^{174} \mathrm{Hf}$ could not be reliably determined as they were populated weakly in the backedtarget data. Unfortunately, no linking transitions or coincidence relationships, consistent with wobbling excitations, were observed between any of the eight SD bands in ${ }^{174} \mathrm{Hf}$. Without such linking transitions, stable triaxiality cannot be experimentally proved at this time for ${ }^{174} \mathrm{Hf}$.

\section{DISCUSSION}

Before we address whether the SD bands in ${ }^{174} \mathrm{Hf}$ show any evidence of triaxiality, it should be noted that SD 1 is nearly isospectral with one of the SD bands observed in ${ }^{175} \mathrm{Hf}$ [14]. This latter structure has been linked to the known levels, and thus the excitation energy $(12.682 \mathrm{MeV})$ as well as the spin/parity $\left(79 / 2^{-}\right)$of the lowest state have been determined. Scholes et al. [14] proposed that this sequence is likely based on a seven-quasiparticle configuration that includes orbitals from the high- $j \pi i_{13 / 2}, v i_{13 / 2}$, and $v j_{15 / 2}$ shells. Identical bands, especially those with large deformation, are often associated with closely related configurations. In particular, they often contain the same high$j$ orbitals [15]. Thus, the strongly deformed bands in ${ }^{174} \mathrm{Hf}$ may well be based on configurations with at least six quasiparticles and be located at high excitation energy $(>10 \mathrm{MeV})$. This is in contrast with the comparatively simple $\pi i_{13 / 2}$ configuration of the TSD bands in the Lu nuclei. One may conclude that the Hf bands are a part of a different "class" of SD bands than the Lu TSD structures [14].

As stated above, if the strongly deformed sequences in ${ }^{174} \mathrm{Hf}$ are triaxial (as suggested by UC calculations), a 

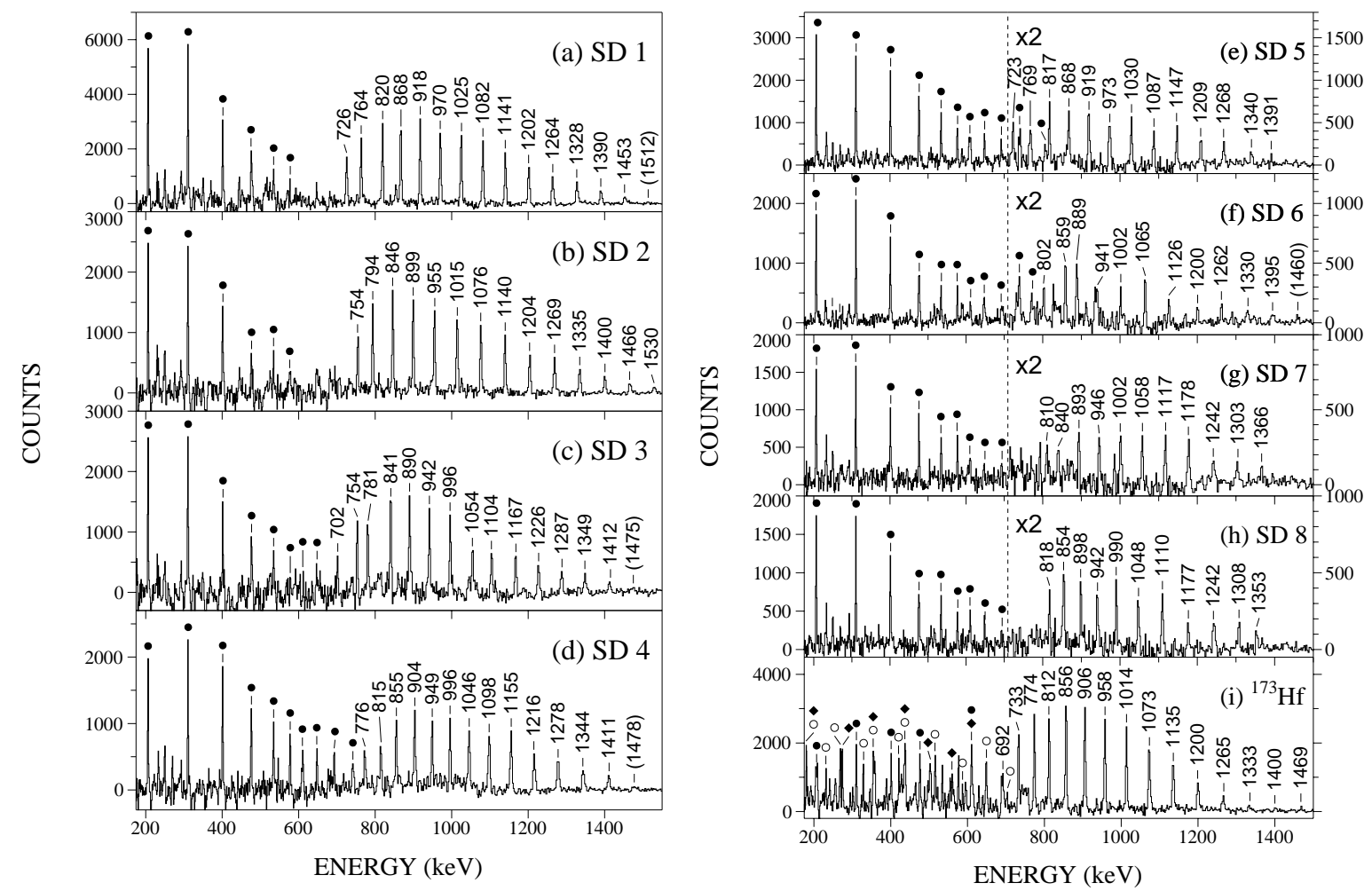

FIGURE 2. Sample spectra for SD bands in ${ }^{174} \mathrm{Hf}$, panels (a)-(h). The spectra were created by summing all possible triple-gate combinations within the hypercube. Transitions denoted with filled circles result from the ground-state band. To the right of the dashed lines in panels (e)-(h), the scale has been reduced by a factor of two. Panel (i) displays the SD band found in ${ }^{173} \mathrm{Hf}$, which was created in the same manner as the other spectra. Transitions denoted with an open circle (filled diamond) are associated with the $v i_{13 / 2}(v[512] 5 / 2)$ band in ${ }^{173} \mathrm{Hf}$. Solid circles in panel (i) are contaminants from ${ }^{174} \mathrm{Hf}$.

family of bands (with nearly identical moments of inertia) are expected to be observed which have different wobbling quanta. It is indeed possible to group the bands in ${ }^{174} \mathrm{Hf}$ into two families based on the behavior with frequency of their moments of inertia, as seen in Fig. 3. Bands 1, 3, 5, and 7 all have nearly the same moments over the observed frequency range [Fig. 3(a)], while bands 2, 6, and 8 [Fig. 3(b)] have moments that are similar to each other, but approximately $10 \%$ less in magnitude. In the context of triaxiality, this could suggest two $n_{w}=0$ configurations associated with bands 1 and 2 (the strongest of the SD sequences) with the weaker structures representing wobbling excitations built on each. The UC predicts TSD minima for all parity/signature combinations, thus, SD 1 and 2 may result from two minima with different parity and/or signature. However, the presence of SD bands displaying similar moments of inertia is not unique to wobbling. Since linking transitions between the SD bands could not be observed, which is a critical signature of wobbling, it is not possible to confirm the presence of triaxiality.

Not only do the present experimental data not prove stable triaxial deformation for ${ }^{174} \mathrm{Hf}$, but they also result in some inconsistencies with the theoretical predictions if triaxiality were present. For example, the measured quadrupole moments are significantly larger than those calculated by the ultimate cranker. The total energy surface (TES) of the $(\pi, \alpha)=(+, 0)$ with $I=50 \hbar$ is shown in Fig. 4. Located near $\varepsilon_{2}=0.25$ and $\gamma=0^{\circ}$, minimum I is representative of the normal deformed configurations. The lowest TSD minimum is labeled as II in Fig. 4, with parameters of $\varepsilon_{2} \approx 0.45$ and $\gamma \approx 27^{\circ}$. A similar minimum is found in each of the parity/signature combinations, and is also observed over a wide spin range $(>25 \hbar)$. These deformations lead to a quadrupole moment of $\sim 9.9 \mathrm{eb}$ that is significantly lower than the experimentally determined values. Since the assertion of triaxiality is based solely on the UC, this large discrepancy is a cause for concern.

A second TSD minimum appears to be located near minimum II and has been labeled IIA in Fig. 4. This minimum has deformation parameters of $\varepsilon_{2} \approx 0.47$ and $\gamma \approx 18^{\circ}$, which suggest a quadrupole moment of $\sim 12.2 \mathrm{eb}$. Perhaps some of the SD bands in ${ }^{174} \mathrm{Hf}$ can be associated with this minimum, since the predicted quadrupole moment lies near 


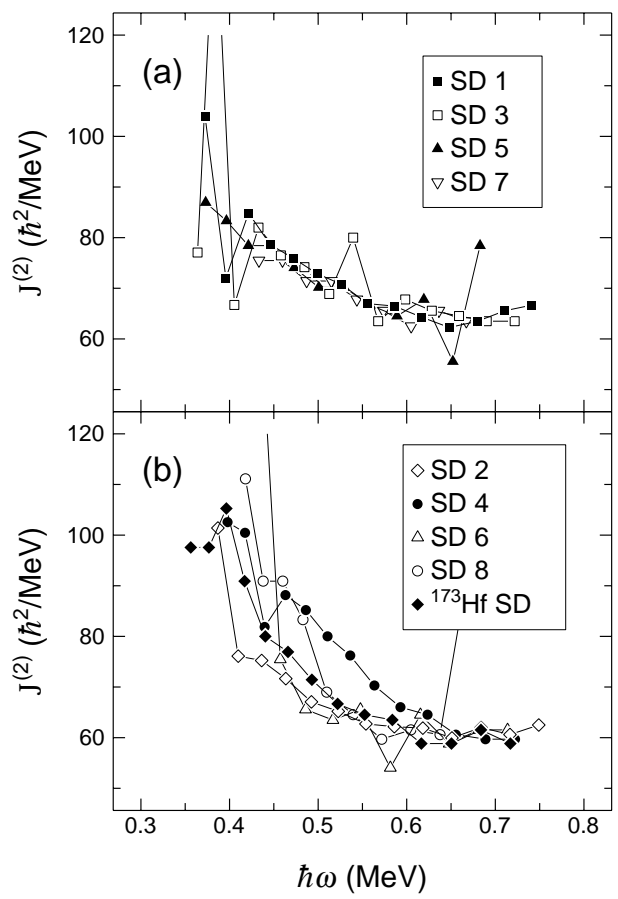

FIGURE 3. The dynamic moments of inertia for the eight SD bands in ${ }^{174} \mathrm{Hf}$ and the one in ${ }^{173} \mathrm{Hf}$. The ${ }^{174} \mathrm{Hf}$ bands were split into possible families of bands based on the similarity of their moments of inertia.

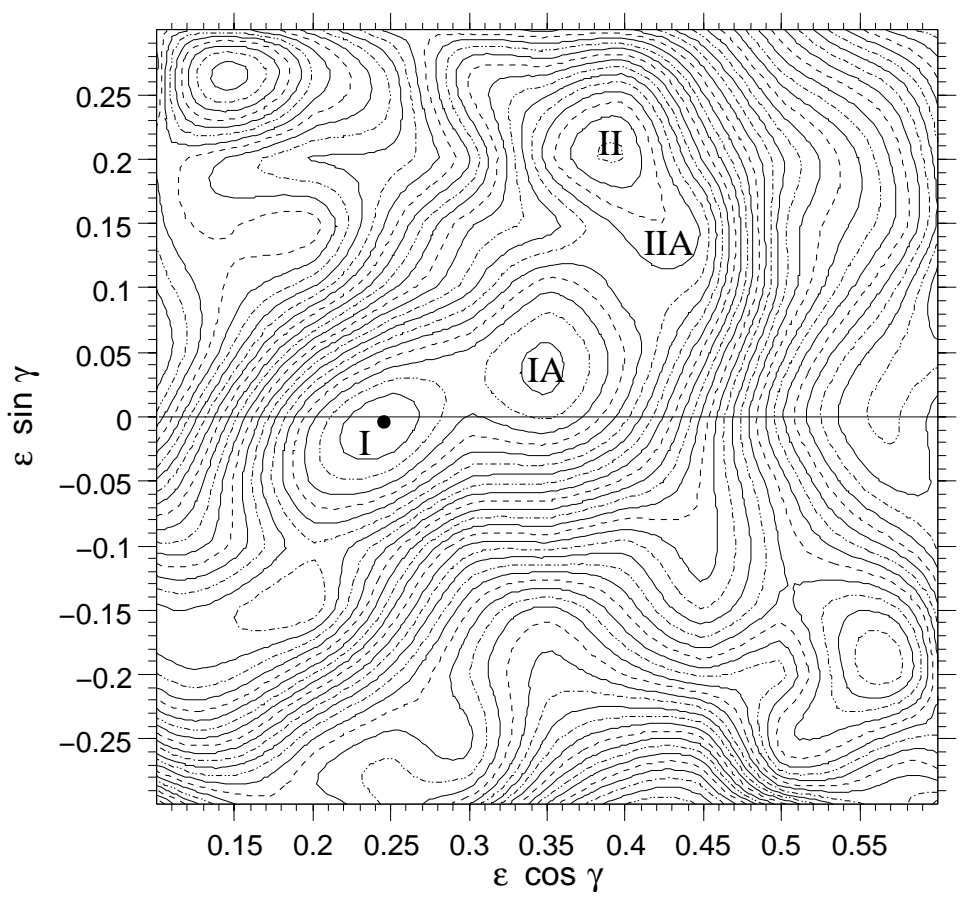

FIGURE 4. Potential energy surface for ${ }^{174} \mathrm{Hf}$ for the lowest $(\pi, \alpha)=(0,+)$ configuration at spin $I=50$. Four minima are marked in the surface and are described in the text. The energy spacing between contour lines is $200 \mathrm{keV}$. 
the determined value. However, in the UC calculations, this minimum is observed over a short spin range $(50-56 \hbar)$ and is only found for the $(\pi, \alpha)=(+, 0)$ combination. One may also consider minimum IA to possibly describe the bands. Similar minima are found in all of the parity/signature combinations with $\varepsilon_{2} \approx 0.35$ and $\gamma \approx 8^{\circ}$. However, the predicted quadrupole moment $(\sim 9.9 \mathrm{eb})$ is again lower than that observed. Therefore, it appears the UC is not able to accurately account for the strongly deformed bands in ${ }^{174} \mathrm{Hf}$ at this time. Further theoretical investigation is necessary to resolve this discrepancy.

It is also interesting to note that only a single SD band is found in the odd- $A$ neighbors ${ }^{173} \mathrm{Hf}$ and ${ }^{175} \mathrm{Hf}[14]$, which is in sharp contrast with the multiplicity of bands seen in ${ }^{174} \mathrm{Hf}$. This observation is not a matter of sensitivity: as can be seen from Fig. 2(i), the candidate SD band in ${ }^{173} \mathrm{Hf}$ was populated with nearly the same strength as SD 1 in ${ }^{174} \mathrm{Hf}$. Since a family of bands was found with SD 1, a family of sequences would also be expected in ${ }^{173} \mathrm{Hf}$ if the strongly deformed structures were indeed triaxial and if a similar energy is associated with the wobbling phonon. If the wobbling excitations were at higher energies in ${ }^{173} \mathrm{Hf}$, however, a lower population could account for their absence. Another possible explanation for the missing bands is that the strongly deformed structure does not lie in a TSD minimum. It seems unlikely, however, that, if a stable triaxial minimum were present, it would not exist in both ${ }^{173} \mathrm{Hf}$ and ${ }^{174} \mathrm{Hf}$.

\section{SUMMARY}

In summary, the measurement of quadrupole moments confirm the large deformation of the four previously known SD bands in ${ }^{174} \mathrm{Hf}$. Four additional, presumably strongly deformed, bands were also observed in ${ }^{174} \mathrm{Hf}$, as well as one in ${ }^{173} \mathrm{Hf}$. However, the non-observation of linking transitions, the discrepancy between experimental and theoretical $Q_{t}$ moments, and the absence of comparable families of bands in ${ }^{173,175} \mathrm{Hf}$ raise serious questions about an interpretation in terms of triaxiality. From the present investigations it is clear that the Hf SD bands are associated with more complex configurations than the TSD bands found in the lighter Lu nuclei. Whether the bands are triaxial or not, the $\mathrm{UC}$ is unable to predict any minimum able to account for the large quadrupole moments found in the data. These new results constitute a considerable challenge for the interpretation of the behavior of $\mathrm{Hf}$ nuclei.

\section{ACKNOWLEDGMENTS}

The authors wish to thank the staff at the 88-Inch and ATLAS accelerator facilities for their support during the experiments. In particular, we thank John Greene for target preparation. Software support by D. C. Radford and H. Jin is gratefully acknowledged. This work was supported by the National Science Foundation under grant Nos. PHY0300673 (USNA) and PHY-0139950 (FSU), as well as the Department of Energy, office of Nuclear Physics, under contract Nos. DE-FG02-96ER40983 (UT), W-31-109-ENG-38 (ANL), and DE-FG02-94ER40848 (UML). D.M.C. acknowledges the receipt of an EPSRC Advanced Fellowship AF/100225.

\section{REFERENCES}

1. A. Bohr and B. R. Mottelson, Nuclear Structure, Vol. 2, Benjamin, New York (1975).

2. G. B. Hagemann and I. Hamamoto, Nucl. Phys. News 13, 20 (2003).

3. S. W. Ødegård et al., Phys. Rev. Lett. 86, 5866 (2001).

4. D. R. Jensen et al., Nucl. Phys. A703, 3 (2002).

5. M. K. Djongolov et al., Phys. Lett. B560, 24 (2003).

6. R. V. F. Janssens and F. S. Stephens, Nucl. Phys. News 6, 9 (1996).

7. M. Cromaz et al., Nucl. Instrum. Methods A462, 519 (2001).

8. K. Starosta et al., Nucl. Instrum. Methods A515, 771 (2003).

9. F. G. Kondev et al., Phys. Rev. C 60, 011303 (1999).

10. H. Amro et al., Phys. Lett. B506, 39 (2001).

11. J. F. Ziegler, J. P. Biersack, and U. Littmark, The Stopping and Range of Ions in Solids (Pergamon, New York, 1985).

12. E. F. Moore et al., Phys. Rev. C 55, R2150 (1997).

13. P. M. Walker, Phys. Scrip. T5, 29 (1983).

14. D. T. Scholes, et al., Phys. Rev. C (to be published).

15. C. Baktash, B. Haas, and W. Nazarewicz, Annu. Rev. Nucl. Part. 